\title{
CXCR4 Antagonist AMD3100 (Plerixafor) Modulates Immune Responses in the Tumor Microenvironment
}

\author{
Ziyao Liu ${ }^{1}$, Jingzhe Wang ${ }^{1}$ and Huabiao Chen ${ }^{2,3,4 *}$ \\ ${ }_{1}^{1}$ Jiangsu Key Laboratory of Clinical Laboratory Medicine, School of Medicine, Jiangsu University, China \\ ${ }^{2}$ Experimental Therapeutics and Molecular Imaging Laboratory, Department of Neurology, Massachusetts General Hospital, USA \\ ${ }^{3}$ Vaccine and Immunotherapy Center, Department of Medicine, Massachusetts General Hospital, USA \\ ${ }^{4}$ Harvard Medical School, Boston, USA
}

Submission: November 13,2020; Published: November 23, 2020

*Corresponding author: Dr. Huabiao Chen, Vaccine and Immunotherapy Center, Massachusetts General Hospital (East), 149 13th Street, Charlestown, MA 02129, USA

\section{Abstract}

AMD3100 (Plerixafor), a specific antagonist of CXCR4, is the most potent small molecule non-peptide inhibitor to CXCR4/CXCL12 axis. The chemokine receptor CXCR4 and its ligand CXCL12 (SDF-1) expressed in a variety of tumor cells play an important role in regulating tumor biological behavior. The tumor microenvironment (TME) is the environment around a tumor, comprising blood vessels, immune cells, fibroblasts, signalling molecules and the extracellular matrix which are involved in tumor growth, invasion, metastasis, immune escape and tumor eradication. Although AMD3100 has been intensively investigated in tumor biology, it remains unclear how this treatment regimen modulates immune cells in the TME, which in turn affects the antitumor efficacy of other therapies. In this review, we specifically revisit the evidence from our and others' studies that AMD3100 acts as an immunomodulator to regulate immune responses in the TME and provide the perspective of synergy of AMD3100 with other therapeutics to prevent tumor development, progression, and metastasis.

Keywords: CXCR4; AMD3100; Plerixafor; Tumor, The tumor microenvironment

\section{Introduction}

Cancer is one of the leading causes of morbidity and mortality worldwide [1]. The high mortality rate of cancer is mainly related to tumor recurrence, metastasis, and lack of effective treatment [2]. Increasing evidence suggests that the interaction between tumor and stromal cells is an integral part of the development and progression of tumors. The tumor microenvironment (TME) is best conceptualized as the composition of a variety of cells, such as noncancerous fibroblasts, adipocytes, immune and vascular cells, as well as signaling molecules and mediators [3-5]. It is gradually recognized that chemokines and their receptors are key communication bridges between tumor cells and stromal cells, creating a favourable microenvironment for tumor growth and metastasis. Chemokine 12 (chemokine 12, CXCL12), also known as stromal cell-derived factor-1 (SDF-1), is commonly expressed in many normal tissue cells and cancer cells [6]. In adult individuals, CXCL12 is involved in different physiological and pathological processes: maintaining tissue balance; participating in the survival and recruitment of immune cells; and promoting the invasion and migration of certain tumor cells $[7,8]$. CXCR4 is a seven-pass transmembrane $\mathrm{G}$ protein-coupled receptor that is highly expressed in a variety of cells and certain tumor cells (such as breast, lung, and prostate cancer) and is associated with early metastasis and poor prognosis [9-11].

Tumor cells with high expression of CXCR4 bind CXCL12expressing stromal cells and thus promote migration and invasion of tumors [7]. Activation of the CXCL12/CXCR4 axis promotes tumor cell proliferation, invasion, distant metastasis and inhibits various signaling pathways of apoptosis [12]. Therefore, the CXCL12/CXCR4 signaling pathway has become a very promising target for tumor therapy $[13,14]$. AMD3100, an antagonist of CXCR4, blocks the signaling of CXCR4 after binding to CXCR4, although cross-reactive to other chemokine receptors as previously reported [15], and thus play an important role in inhibition of tumor growth and metastasis. 


\section{Cancer Therapy \& Oncology International Journal}

Emerging evidence demonstrates that the accumulation of immunosuppressive cells in the TME greatly reduce antitumor efficacy of the treatment, making it one of the major obstacles to cancer immunotherapy [16,17]. Immune cells are involved in different periods of tumor progression. In the initial stages of tumor development, macrophages directly kill tumor cells to promote antitumor responses and indirectly recruit and activate strong infiltration of other immune cells [18]. An intense infiltration of CD8+ T cells is usually associated with a good prognosis [19]. The presence of M2-polarized macrophages is widely recognized as a marker of poor prognosis [20,21]. Therefore, an important cancer treatment strategy is to regroup and redirect immune responses in the TME for a long-term elimination of tumor cells [22-24].
In this review, we focus on the regulation of immune cells by AMD3100 in the TME and detail four types of immune cells: CD8+ $\mathrm{T}$ cells, regulatory $\mathrm{T}$ cells (Tregs), tumor-associated macrophages (TAMs), and myeloid-derived suppressor cells (MDSCs).

\section{Effects of AMD3100 on immune cells in the TME}

The occurrence and development of tumors are inseparable from the TME. AMD3100 not only directly targets the CXCR4/ CXCL12 axis to inhibit tumor growth and metastasis but also acts as a potent immunomodulator to enhance antitumor immune responses and prevent the development of a multi-faceted immunosuppressive intratumoral microenvironment (Figure 1).

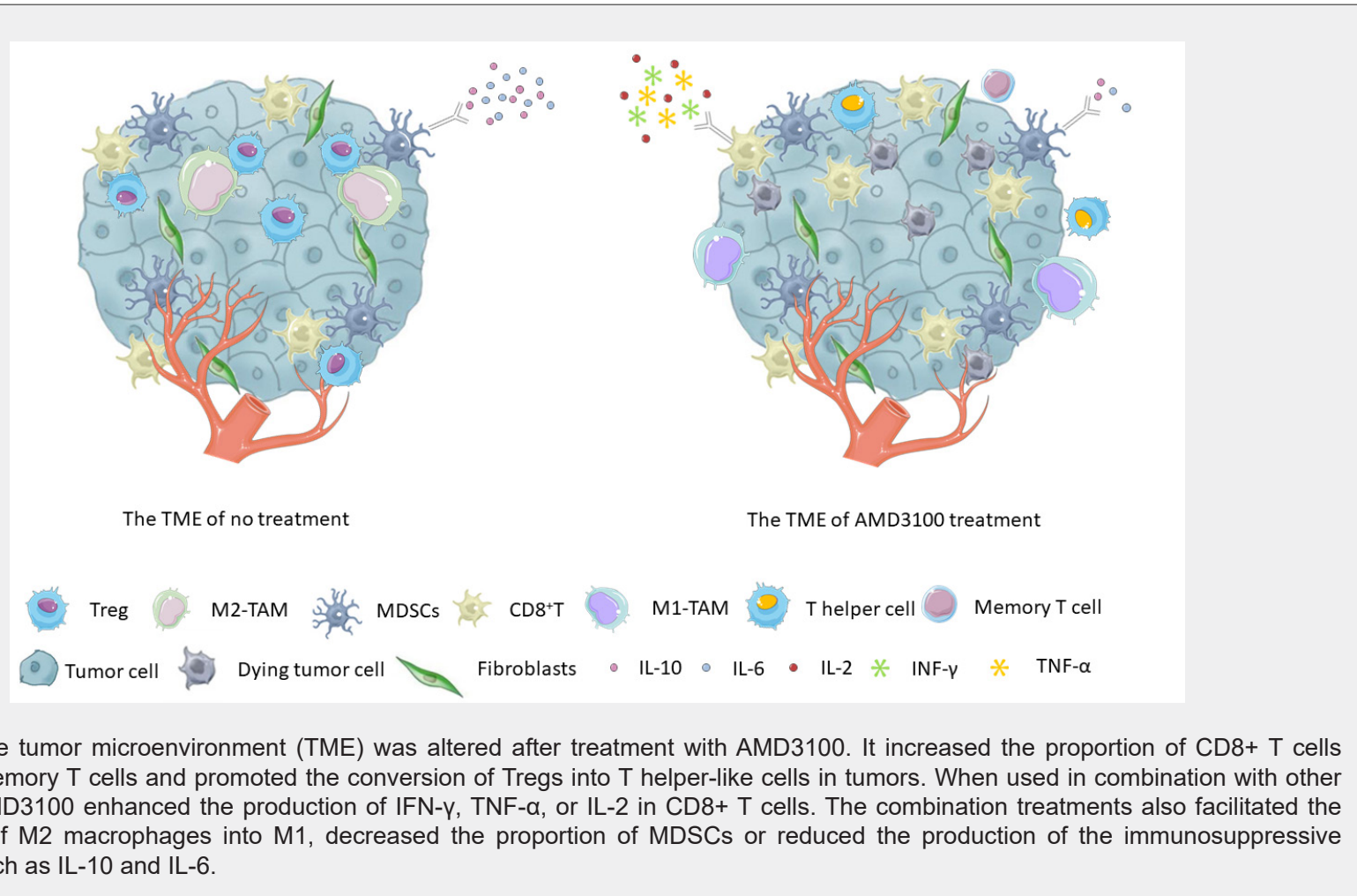

\section{CD8+ T cells}

In the TME, CD8+ T cells play an important role in antitumor immunity. CD8+ $\mathrm{T}$ cells differentiate into cytotoxic $\mathrm{T}$ cells (CTLs) and exhibit cytotoxicity against tumor cells. Terminally differentiated effector CD8+ T cells are IL-2 dependent and highly cytotoxic [25]. The overall survival rate of high-grade gliomas (HGG) was associated with the changes of tumor-infiltrating CD8+ T cells during treatment. AMD3100 in combination with bevacizumab resulted in a significant increase of CD8+ T cells [26]. In a pancreatic ductal adenocarcinoma (PDA) model, AMD3100 induced rapid accumulation of $\mathrm{T}$ cells in tumors and synergized with $\alpha$ PD-L1 to produce a significant reduction of tumors [27]. In addition, Zeng $\mathrm{Y}$ et al. found that combination therapy with AMD3100 and $\alpha$ PD-1 further increased the infiltration of CD8+
T cells in ovarian tumors compared to $\alpha$ PD-1 monotherapy [28]. Some evidence suggests that AMD3100 increases the proportion of CD8+ memory T cells and enhances antitumor activity [28,29]. Furthermore, AMD3100 monotherapy and AMD3100-VIC-008 combination therapy inhibited PD-1 expression on CD8+ T cells in spleens, lymph nodes, and tumors of a mouse model of mesothelioma [30]. AMD3100, when combined with other therapies, significantly enhanced the expression of IFN- $\gamma$, TNF- $\alpha$ or IL-2 in CD8+ T cells [28,31].

\section{Regulatory T cells}

Regulatory $\mathrm{T}$ cells (Tregs) are immunosuppressive cells in lymphoid immune cells that interfere with antigen presentation by tumor cells and help tumor cells achieve immune escape. They are in a low number in normal tissue but significantly increase 
in many tumor tissues. Blocking the migration of Tregs into the TME is a promising approach for tumor immunotherapy [32]. Allogeneic hematopoietic cell transplantation (AlloHCT) has been supported as an effective treatment option for patients with refractory or relapsed non-Hodgkin's lymphoma. Dürr C et al. elucidated that CXCR4 blockade not only reduced Treg migration into tumor tissue, but also enhanced the antitumor response after AlloHCT [33]. In addition, blocking CCL3-CCR1/CCR5 and CXCL12-CXCR4 axes inhibited Treg accumulation in the leukemic hematopoietic microenvironment (LHME) and delayed leukemia development [32]. Righi E et al. first revealed that AMD3100 treatment impaired Treg migration in vitro and reduced CD4+ CD25+ and CD4+ CD25+ FoxP3+ T cells in vivo [34]. Li B et al. further reported that Tregs were converted to IL2+ CD40L+ $\mathrm{T}$ helper-like cells and might lose their immunosuppressive function after the combined treatment with AMD3100 and VIC008 [30]. Following this study, Zeng Y et al. further intensively studied the effect of AMD3100 on Treg cells and found that combination therapy with $\alpha$ PD- 1 significantly inhibited Treg cells in tumors and increased the proportion of CD4+CD25- Foxp3+ T cells, phenotypically characteristic as CD40L+ IL-2+ T helper-like cells [28].

\section{Tumor-associated macrophages}

Tumor-associated macrophages (TAMs) are the most predominant and highly infiltrating cells in the TME [35]. TAMs regulate immune cell recruitment and inhibits antitumor responses by producing growth factors, chemokines and cytokines and resulting in an immunosuppressive TME [36]. TAMs are categorized as M1 or M2 types. M1 expresses proteins such as tumor necrosis factor (TNF), inducible nitric oxide lyase and MHCII, which mainly enhance anti-tumor. M2 highly expresses CD163, CD204 and CD206, which promote tumor development [37]. AMD3100 has been demonstrated to inhibit M2 accumulation by blocking CXCR4/SDF-1 signaling [38]. The upregulation of CXCR4 in radioresistant tumor ECs is highly correlated with SDF-1TAM recruitment and TAM M2 polarization [39]. Recently, Zeng Yang et al. showed that AMD3100 in combination with $\alpha \mathrm{PD}-1$ reduced inhibitory leukocytes and promoted polarization to M1 from M2 macrophages in tumors in an ovarian cancer model [28].

\section{Myeloid-derived suppressor cells}

Myeloid-derived suppressor cells (MDSCs) are a group of immature bone marrow-derived cells with immunosuppressive functions. MDSC accumulation in tumors promotes immune escape [40,41]. Osteosarcoma tissues were infiltrated with many MDSCs, which inhibit the expansion of CTLs. The majority of tumor-infiltrating MDSCs were CXCR4 positive and migrate toward the SDF-1 gradient. Binding of SDF-1 to its receptor CXCR4 activates the downstream AKT pathway, resulting in reduced apoptosis of MDSCs. And on this basis, AMD3100 promoted
CD8+ T cell expansion by reducing CXCR4-expressing MDSCs in K7M2 osteosarcoma tumors [42]. The combined treatment with AMD3100 and $\alpha$ PD-1 significantly decreased the proportion of MDSCs and reduced the production of immunosuppressive cytokines IL-10 and IL-6, which might decrease the immunosuppressive effect [28]. Transmembrane TNF- $\alpha$ promotes the recruitment of MDSCs to tumor tissues by up-regulating the expression of CXCR4, but this can be partially blocked by treatment with the CXCR4 inhibitor AMD3100 [40]. The infiltration of MDSCs into the metastatic liver tumors may be mediated by up regulating the expression of CXCR4, and AMD3100 blocks the interaction of tumor and stromal cells in the metastatic cascade to the liver [43]. Estrogen plays an important role in the occurrence, metastasis, and drug resistance of estrogen receptor (ER) positive breast cancer. Ouyang L et al. reported that after treatment with estrogen high levels of SDF- $1 \alpha$ and tumor-infiltrating MDSCs were detected while blockade of the SDF-1/CXCR4 axis with AMD3100 neutralized the effect of estrogen on tumorigenesis [44].

\section{Perspective}

The application of AMD3100 in cancer treatment has not been well characterized, particularly in AMD3100-mediated immune modulation. While effector $\mathrm{T}$ cells execute antitumor activities and eliminate tumor cells, immunosuppressive cells participate in tumor immune escape through various mechanisms. In-depth study of immune cells in the TME can provide useful diagnosis, prediction, and prognostic information. The research of AMD3100 on the regulation of immune cells has profound significance and lays a solid foundation for future research and treatment, highlighting AMD3100 working synergistically with other therapeutics to enhance the antitumor efficacy.

\section{References}

1. Woodall MJ, Neumann S, Campbell K, et al. The Effects of Obesity on Anti-Cancer Immunity and Cancer Immunotherapy. Cancers (Basel) 12(5): E1230.

2. Mortezaee K (2020) CXCL12/CXCR4 axis in the microenvironment of solid tumors: A critical mediator of metastasis. Life Sci 249: 117534.

3. Dominiak A, Chełstowska B, Wioletta Olejarz, Grażyna Nowicka (2020) Communication in the Cancer Microenvironment as a Target for Therapeutic Interventions. Cancers (Basel) 12(5): E1232.

4. Galland S, Martin P, Fregni G, Igor Letovanec, Ivan Stamenkovic (2020) Attenuation of the pro-inflammatory signature of lung cancer-derived mesenchymal stromal cells by statins. Cancer Lett 484(1): 50-64.

5. Lee SC, Dacheux MA, Norman DD, Louisa Balázs, Raul M Torres, et al. (2020) Regulation of Tumor Immunity by Lysophosphatidic Acid. Cancers (Basel) 12(5): E1202.

6. Floranović MP, Veličković LJ (2019) Effect of CXCL12 and Its Receptors on Unpredictable Renal Cell Carcinoma. Clin Genitourin Cancer 18(4): e337-e342.

7. Pozzobon T, Goldoni G, Viola A, Barbara Molon (2016) CXCR4 signaling in health and disease. Immunol Lett 177: 6-15. 


\section{Cancer Therapy \& Oncology International Journal}

8. Döring Y, Noels H, van der Vorst EPC, Carlos Neideck, Virginia Egea, et al. (2017) Vascular CXCR4 Limits Atherosclerosis by Maintaining Arterial Integrity: Evidence from Mouse and Human Studies. Circulation 136(4): 388-403.

9. Krikun G (2018) The CXL12/CXCR4/CXCR7 axis in female reproductive tract disease: Review. Am J Reprod Immunol 80(5): e13028.

10. Jäger B, Klatt D, Plappert L, Heiko Golpon, Stefan Lienenklaus, et al (2020) CXCR4/MIF axis amplifies tumor growth and epithelialmesenchymal interaction in non-small cell lung cancer. Cell Signal 73: 109672.

11. Walenkamp AME, Lapa C, Herrmann K, Hans-Jürgen Wester, et al (2017) CXCR4 Ligands: The Next Big Hit? J Nucl Med 58 (Suppl 2): 77S-82S.

12. Ullah TR (2019) The role of CXCR4 in multiple myeloma: Cells' journey from bone marrow to beyond. J Bone Oncol 17: 100253.

13. Jiang J, Shen N, Song W, Haiyang Yu, Kazuo Sakurai, et al. (2019) Combretastatin A4 nanodrug combined plerixafor for inhibiting tumor growth and metastasis simultaneously. Biomater Sci 7(12): 52835291.

14. Wang J, Tannous BA, Poznansky MC, Chen H (2020) CXCR4 antagonist AMD3100 (plerixafor): From an impurity to a therapeutic agent. J Pharmacol Res 159: 105010.

15. Wald O (2018) CXCR4 based therapeutics for Non-Small Cell Lung Cancer (NSCLC). J Clin Med 7(10): 303.

16. Sun B, Hyun H, Li LT, Andrew Z Wang (2020) Harnessing nanomedicine to overcome the immunosuppressive tumor microenvironment. Acta Pharmacol Sin 41(7): 970-985.

17. Li Z, Wang Y, Shen Y, Chenggen Qian, David Oupicky, et al. (2020) Targeting pulmonary tumor microenvironment with CXCR4-inhibiting nanocomplex to enhance anti-PD-L1 immunotherapy. Sci Adv 6(20): eaaz 9240 .

18. Lopez YM, Cassetta L, Pollard JW (2020) Macrophage targeting in cancer. Ann N Y Acad Sci.

19. Pagès F, Mlecnik B, Marliot F, Gabriela Bindea, Fang Shu Ou, et al. (2018) International validation of the consensus Immunoscore for the classification of colon cancer: a prognostic and accuracy study. Lancet 391(10135): 2128-2139.

20. Petiprez F, Meylan $M$, de Reyniès $A$, Catherine Sautès-Fridman, Wolf $H$ Fridman, et al. (2020) The Tumor Microenvironment in the Response to Immune Checkpoint Blockade Therapies. Front Immunol 11: 784

21. Lee NY, Kim Y, Kim YS, Yuri Kim (2020) $\beta$-Carotene exerts anti-colon cancer effects by regulating M2 macrophages and activated fibroblasts. J Nutr Biochem 82: 108402.

22. Guo F, Wang Y, Liu J, S C Mok, F Xue, et al. (2016) CXCL12/CXCR4: a symbiotic bridge linking cancer cells and their stromal neighbors in oncogenic communication networks. Oncogene 35(7): 816-826.

23. Mortezaee K (2020) CXCL12/CXCR4 axis in the microenvironment of solid tumors: A critical mediator of metastasis. Life Sci 249: 117534

24. Do HTT, Lee CH, Cho J (2020) Chemokines and their Receptors: Multifaceted Roles in Cancer Progression and Potential Value as Cancer Prognostic Markers. Cancers (Basel) 12(2): 287.

25. Iwahori K (2020) Cytotoxic CD8 Lymphocytes in the Tumor Microenvironment. Adv Exp Med Biol 1224: 53-62.

26. Lee EQ, Duda DG, Muzikansky A, Elizabeth R Gerstner, John G Kuhn, et al. (2018) Phase I and Biomarker Study of Plerixafor and Bevacizumab in Recurrent High-Grade Glioma. Clin Cancer Res 24(19): 4643-4649.
27. Garg B, Giri B, Modi S, Vrishketan Sethi, Iris Castro, et al. (2018) NFкB in Pancreatic Stellate Cells Reduces Infiltration of Tumors by Cytotoxic $\mathrm{T}$ Cells and Killing of Cancer Cells, via Up-regulation of CXCL12. Gastroenterology 155(3): 880-891.e8.

28.Zeng Y, Li B, Liang Y, Patrick M Reeves, Xiying Qu, et al. (2019) Dual blockade of CXCL12-CXCR4 and PD-1-PD-L1 pathways prolongs survival of ovarian tumor-bearing mice by prevention of immunosuppression in the tumor microenvironment. Faseb J 33(5): 6596-6608.

29. Vianello F, Papeta N, Chen T, , Paul Kraft, Natasha White, et al. (2006) Murine B16 melanomas expressing high levels of the chemokine stromal-derived factor-1/CXCL12 induce tumor-specific $\mathrm{T}$ cell chemorepulsion and escape from immune control. J Immunol 176(5): 2902-2914.

30. Li B, Zeng Y, Reeves PM, Chongzhao Ran, Qiuyan Liu, et al. (2018) AMD3100 Augments the Efficacy of Mesothelin-Targeted, ImmuneActivating VIC-008 in Mesothelioma by Modulating Intrarumoral Immunosuppression. Cancer Immunol Res 6(5): 539-551.

31. Gaugler B, Arbez J, Legouill S, Pierre Tiberghien, Philippe Moreau, et al (2013) Characterization of peripheral blood stem cell grafts mobilized by granulocyte colony-stimulating factor and plerixafor compared with granulocyte colony-stimulating factor alone. Cytotherapy 15(7): 861-868.

32. Wang R, Feng W, Wang H, Lina Wang' Xiao Yang, et al. (2020) Blocking migration of regulatory $\mathrm{T}$ cells to leukemic hematopoietic microenvironment delays disease progression in mouse leukemia model. Cancer Lett 469: 151-161.

33. Dürr C, Pfeifer D, Claus R, Annette Schmitt-Graeff, Ulrike V Gerlach et al. (2010) CXCL12 mediates immunosuppression in the lymphoma microenvironment after allogeneic transplantation of hematopoietic cells. Cancer Res 70 (24): 10170-10781.

34. Righi E, Kashiwagi S, Yuan J, Michael Santosuosso, Pierre Leblanc, et al. (2011) CXCL12/CXCR4 blockade induces multimodal antitumor effects that prolong survival in an immunocompetent mouse model of ovarian cancer. Cancer Res 71(16): 5522-5534.

35. Yang W, Yang S, Zhang F, Feng Cheng, Xuehao Wang, et al. Influence of the Hippo-YAP signalling pathway on tumor associated macrophages (TAMs) and its implications on cancer immunosuppressive microenvironment. Ann Transl Med 8(6): 399.

36. Fu LQ, Du WL, Cai MH, Jia-YuYao, Yuan-Yuan Zhao, et al. (2020) The roles of tumor-associated macrophages in tumor angiogenesis and metastasis. Cell Immunol 353: 104119.

37. Childs RW, Carlsten M (2015) Therapeutic approaches to enhance natural killer cell cytotoxicity against cancer: the force awakens. Nat Rev Drug Discov 14(7): 487-498.

38. Brenner C, Franz WM, Kühlenthal S, F Remm, L Gross, et al. (2015) DPP-4 inhibition ameliorates atherosclerosis by priming monocytes into M2 macrophages. Int J Cardiol 199: 163-169.

39. Choi SH, Kim AR, Nam JK, Jin-Mo Kim, Jee-Youn Kim, et al. (2018) Tumour-vasculature development via endothelial-to-mesenchymal transition after radiotherapy controls CD44v6 cancer cell and macrophage polarization. J Nat Commun 9: 5108

40. Choi Seo-Hyun, Kim A-Ram, Nam Jae-Kyung, Jin-Mo Kim, Jee-Youn Kim, et al. (2018) Tumour-vasculature development via endothelial-tomesenchymal transition after radiotherapy controls CD44v6 cancer cell and macrophage polarization. J Nat Commun 9(1): 5108.

41. Wang Y, Jia A, Bi Y, Yue Xin Wang, Guangwei Liu, et al. (2020) Metabolic Regulation of Myeloid-Derived Suppressor Cell Function in Cancer Cells 9(4): 1011. 


\section{Cancer Therapy \& Oncology International Journal}

42. Jiang K, Li J, Zhang J, Lei Wang, Qianfeng Zhang, et al. (2019) SDF-1/ CXCR4 axis facilitates myeloid-derived suppressor cells accumulation in osteosarcoma microenvironment and blunts the response to antiPD-1 therapy. Int Immunopharmacol 75: 105818.

43. Benedicto A, Romayor I, Arteta B (2018) CXCR4 receptor blockage reduces the contribution of tumor and stromal cells to the metastatic growth in the liver. Oncol Rep 39(4): 2022-2030.

44. Ouyang L, Chang W, Fang B, Jieting Qin, Xincai Qu, et al. (2016) Estrogen-induced SDF- $1 \alpha$ production promotes the progression of ER-negative breast cancer via the accumulation of MDSCs in the tumor microenvironment. Sci Rep 6: 39541.

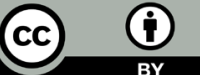

This work is licensed under Creative Commons Attribution 4.0 License DOI:10.19080/CTOIJ.2020.17.555962
Your next submission with Juniper Publishers will reach you the below assets

- Quality Editorial service

- Swift Peer Review

- Reprints availability

- E-prints Service

- Manuscript Podcast for convenient understanding

- Global attainment for your research

- Manuscript accessibility in different formats ( Pdf, E-pub, Full Text, Audio)

- Unceasing customer service

Track the below URL for one-step submission https://juniperpublishers.com/online-submission.php 